\title{
Regioselective metal deposition on polymer-Au nanoparticle hybrid chains
}

\author{
Zhiqi Huang ${ }^{1}$, Sihang Liu ${ }^{1}$, Yuejiao Zhang ${ }^{3}$, Jingliang Yang ${ }^{3}$, Jianfeng $\mathrm{Li}^{3}$, Zhihong $\mathrm{Nie}^{2^{*}}$ and \\ Jinlong Gong ${ }^{1 *}$
}

Templated growth is an important synthetic strategy for the construction of complex multicomponent nanostructures [1-3]. The commonly-used templates can be made from either "hard" materials [4-7] (e.g., silica) or "soft" materials (e.g., micelles [8], polymers [9], supramolecules [10], etc.). Hard templates typically allow for good crystallinity of the final nanostructures and better preservation of the geometric feature of original templates $[7,11]$, while soft templates are relatively easy to prepare and remove during the synthesis [12,13]. Recently, polymer-tethered inorganic nanoparticle (NP) assemblies (e.g., one-dimensional chains [14-17], two-dimensional sheets [18], and three-dimensional vesicles [19]) have attracted reasonable attention for their potential applications in diverse fields, such as bio-imaging and drug delivery [16] and surface enhanced Raman scattering (SERS) [20]. Furthermore, these polymer-inorganic NP assemblies can serve as a new class of mixed soft-hard colloidal template for the fabrication of unconventional nanostructures. For instance, Nie and co-authors [21] firstly synthesized a variety of coaxial-like and Saturn-like multicomponent nanostructures by selective deposition of metals (e.g., Pd, Pt, Ag, Ni, etc.) or metal oxides (e.g., $\mathrm{CeO}_{2}$ and $\left.\mathrm{Cu}_{2} \mathrm{O}\right)$ on seed Au NPs organized in polymer$\mathrm{Au} \mathrm{NP}$ assemblies, followed by dissociation of the assemblies. Nevertheless, there remains a challenge to precisely control the selective deposition of growth materials on the mixed soft-hard templates.

In this paper, we report the regioselective deposition of metals on alternatively arranged polymer-Au NP hybrid chains (HCs). By varying the surfactants and hence thermodynamics of metal nucleation and growth, sec- ondary metals (e.g., Pt, Pd, Au, etc.) could be deposited either solely on the surface of Au NPs (hard templates) to produce alternatively-coated HCs (AHCs) or over the entire surface of HCs (both hard Au NPs and soft polymer domains) to form peapod-like HCs (PHCs) (Fig. 1a). The composition and position of metal coatings were found to drastically alter the optical response of the hybrid nanostructures. Both AHCs and PHCs with Pt shell led to the damping of the localized surface plasmon resonance (LSPR). In contrast, homogeneous Au shell of PHCs exhibited a strong plasmon coupling between the inner Au NPs and outer shell, as confirmed by the finitedifference time-domain (FDTD) simulations. When used as SERS substrates, the Au-shelled PHCs showed significantly enhanced Raman signal which was 6.6 and 2.1 times higher than that of Pt-shelled PHCs and Pt-shelled AHCs, respectively.

Polymer-Au NP HCs composed of Au NPs ( $20 \mathrm{~nm}$ in diameter) and nanoscale polystyrene (PS) domains were prepared and transferred into aqueous solution according to our previous report [21] (see detailed procedures in Supplementary information (SI)). In brief, Au NPs were first surface-modified with thiol-terminated polystyrene (PS-SH, $\left.M_{\mathrm{n}}=12,000\right)$ and redispersed in dimethylformamide (DMF). A mixture solvent of DMF/water was then added drop-wise into the NP dispersion to initiate their self-assembly into HCs with linear arrangement of tens of Au NPs. The HCs were dialyzed against water to remove DMF and collected by gentle centrifugation. The alternating arrangement of PS and Au domains of the HCs could be clearly observed and distinguished with transmission electron microscope (TEM): the hard Au NPs are

\footnotetext{
${ }^{1}$ Key Laboratory for Green Chemical Technology of Ministry of Education, School of Chemical Engineering and Technology, Tianjin University, Collaborative Innovation Center of Chemical Science and Engineering, Tianjin 300072, China

${ }^{2}$ State Key Laboratory of Molecular Engineering of Polymers, Department of Macromolecular Science, Fudan University, Shanghai 200438, China

${ }^{3}$ State Key Laboratory for Physical Chemistry of Solid Surfaces, MOE Key Laboratory of Spectrochemical Analysis and Instrumentation, College of Chemistry and Chemical Engineering, iChEM, Xiamen University, Xiamen 361005, China

* Corresponding authors (emails: znie@fudan.edu.cn (Nie Z); jlgong@tju.edu.cn (Gong J))
} 

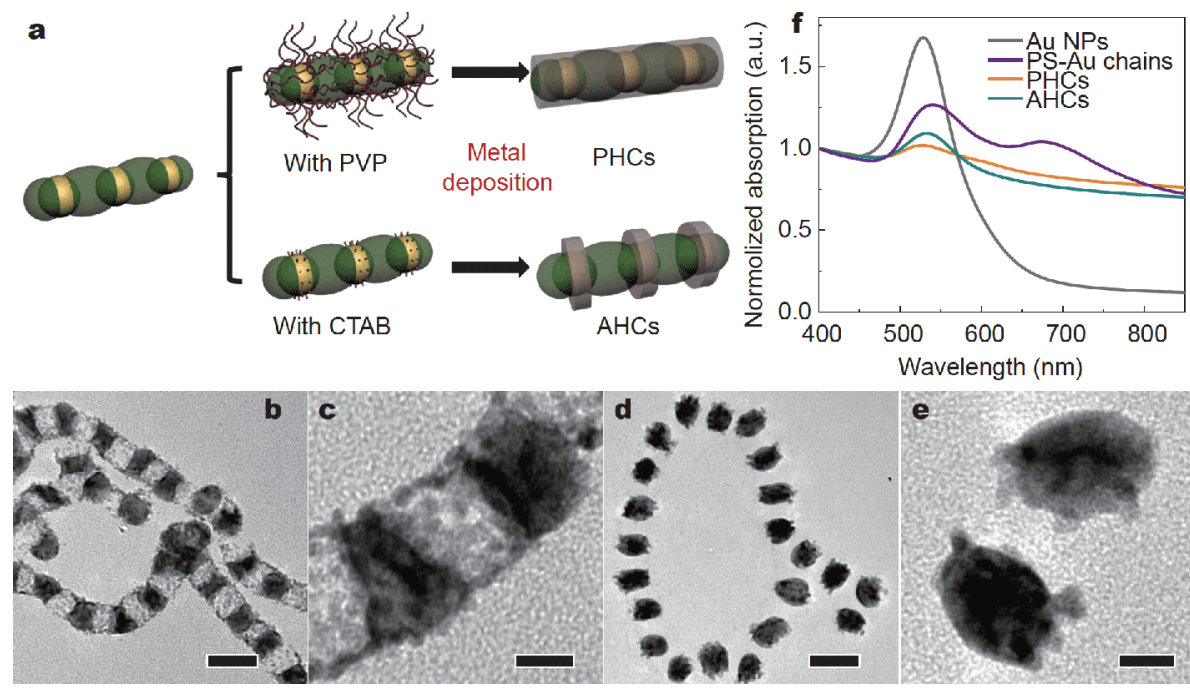

Figure 1 (a) Schematic illustration of controlled metal deposition on PS-Au NP HCs to produce either PHCs or AHCs. (b) TEM and (c) HRTEM images of Pt-coated PHCs. (d) TEM and (e) HRTEM images of Pt-coated AHCs. Scale bars are $50 \mathrm{~nm}$ in (b, d) and $20 \mathrm{~nm}$ in (c, e). (f) UV-vis absorption spectra of Au NPs, original PS-Au HCs, Pt-coated PHCs, and Pt-coated AHCs.

dark, while the soft PS blocks are light grey (Fig. S1a). The distance between adjacent Au NPs in the chains (i.e., the length of PS domains along the chain direction) is $13 \mathrm{~nm}$, which can be tuned by varying the length of PS chains (Fig. S1b).

The HCs were used as templates for the further coating of a second metal (e.g., Pt, Pd, Au, etc.) in an aqueous solution containing metal precursor and surfactant (Fig. 1a). Ascorbic acid was subsequently added as a reducing agent and the mixture was incubated for $10 \mathrm{~h}$ to complete the reaction. The selective metal deposition was achieved by appropriate surfactants. When polyvinylpyrrolidone (PVP, $M_{\mathrm{w}}=29,000$ ) was used as the surfactant, metal deposition occurred homogeneously over the entire HCs to produce PHCs. In contrast, the use of cetyltrimethylammonium bromide (CTAB) as the surfactant led to the formation of AHCs with selective metal coating solely on the hard Au NPs.

Pt was chosen to demonstrate the selective metal deposition. As shown in Fig. 1b, c, after Pt deposition, a thin porous Pt skin covers both the exposed Au NP surface and the PS domains of the PHCs, due to the autocatalyzed growth nature of Pt [21]. This Pt skin in return helps to outline the shape of PS blocks which show relatively low contrast under TEM. Whereas in the case of AHCs, the nucleation and growth of Pt exclusively occur on the exposed Au NP surfaces, with no Pt skin on the surface of PS blocks (Fig. 1d, e). The different coating also leads to various absorption features of the resulting nanostructures (Fig. 1f). The original PS-Au NP HCs show a strong absorption peak at $540 \mathrm{~nm}$ arising from transverse LSPR of Au NPs and a weak peak at $680 \mathrm{~nm}$ due to longitudinal interparticle plasmonic coupling [22]. It is known that Pt coating hinders the excitation of surface plasmon, leading to the LSPR damping. Upon Pt coating, the LSPR peak at $540 \mathrm{~nm}$ significantly weakened for both PHCs and AHCs due to the damping effect of Pt shell [23]. What's more, the disappearance of LSPR at $680 \mathrm{~nm}$ suggests that longitudinal coupling becomes negligible on account of the LSPR damping. PHCs with the thorough Pt coating suffer a stronger effect of LSPR damping than AHCs.

To better understand the critical role of PVP, we systematically varied the concentration of PVP in the growth solution, while keeping all other parameters constant. When there was no addition of PVP, Pt was only selectively deposited on Au NPs to produce AHCs (Fig. 2a). Pt deposition on PS blocks started to occur with the addition of $0.1 \mathrm{wt} \%$ of PVP in the growth solution (Fig. 2b). This phenomenon became predominant with the increase in the concentration of PVP, evidenced by the increased accumulation of Pt on PS blocks (Fig. 2c, d). We note that the chain length of PVP do not affect the formation of PHCs. At a given PVP concentration, PVP with much shorter chain length $\left(M_{\mathrm{w}}=10,000\right)$ also led to the formation of PHCs (Fig. S2). The PVP chains would adsorb or wrap on the PS-Au NP chains to lower the interfacial energy between hydrophobic PS blocks and water [24,25], thus promoting the nucleation and growth of $\mathrm{Pt}$ on the whole chain. With PVP, Pt can be deposited onto Au@PS 


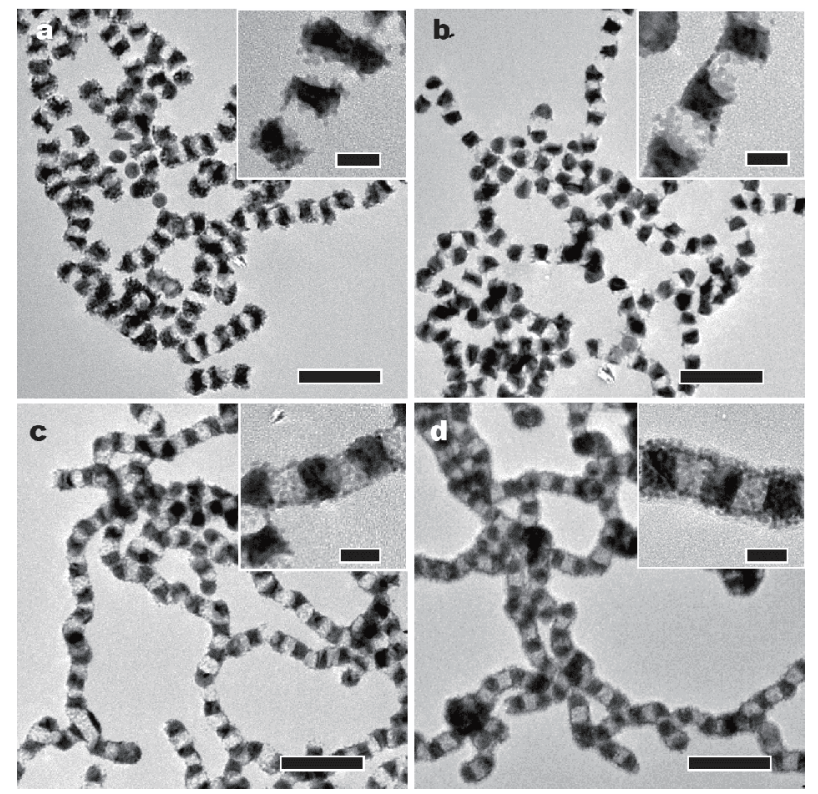

Figure 2 TEM images of Pt-shelled PHCs obtained with different concentrations of PVP: (a) $0 \mathrm{wt} \%$, (b) $0.1 \mathrm{wt} \%$, (c) $0.5 \mathrm{wt} \%$ and (d) $1 \mathrm{wt} \%$. Insets are the high resolution TEM images highlighting the $\mathrm{Pt}$ deposition on PS blocks. Scale bars are $100 \mathrm{~nm}$ in (a-d) and $20 \mathrm{~nm}$ in insets.

core-shell unimicells, forming Au@PS@Pt multi-shell nanospheres (Fig. S3).

Such selective metal deposition is independent on kinetic factors such as precursor amount. Our previous research has pointed out that in presence of CTAB, small amount of precursor led to exclusive nucleation and growth on NPs, while overgrowth of metals on PS block may occur with the addition of large amount of precursor [21]. However, when PVP was added, the metal could be deposited onto PS blocks even when the amount of precursor was extremely small $(0.001 \mathrm{mmol})$ (Fig. S4).

This approach can be applied for the selective deposition of a variety of metals. The controlled deposition of Pd led to the formation of both Pd-shelled PHCs and Pdcoated AHCs (Fig. 3a, b). However, when Au or Ag was used, the deposition was always limited on Au NPs to yield AHCs, with any concentration of PVP in the growth solution (Fig. 3c and Fig. S5). This is due to the fact that epitaxial growth of $\mathrm{Au}$ on $\mathrm{Au}$ leads to a much less increase in the interfacial energy than the deposition of $\mathrm{Au}$ on PVP-covered PS domains [21,26]. Nevertheless, we could still manage to achieve the coating of $\mathrm{Au}$ onto Ptshelled PHCs to obtain Au/Pt-shelled PHCs. TEM images in Fig. 3d show that these Au/Pt-shelled PHCs have denser shell with increased thickness of $5.1 \pm 0.9 \mathrm{~nm}$ (Fig. S6), compared with Pt-PHCs (2.2 \pm 0.4 nm, Fig. S7).



Figure 3 (a, b) TEM images of Pd-shelled (a) AHCs and (b) PHCs. (c, d) TEM images of Au-shelled (c) AHCs and (d) PHCs. The bottom-left insets in $(c, d)$ show the corresponding structural model of nanostructures. The insets are high resolution TEM images highlighting the Pt deposition on PS blocks. Scale bars are $50 \mathrm{~nm}$ in (a-d) and $20 \mathrm{~nm}$ in insets.

In addition to the major SPR peak at $540 \mathrm{~nm}$, the multiple plasmonic coupling between the Au shell and inner $\mathrm{Au}$ NP core in the PHCs resulted in two weak LSPR peaks located at 615 and $925 \mathrm{~nm}$, respectively (Fig. S8).

The electromagnetic field distributions within Ptshelled PHCs and Au/Pt-shelled PHCs were computed via Finite-difference time-domain (FDTD) simulation based on the structural model in Fig. S9 (see simulation detail in ESI). The results indicate that the Pt-PHCs and Au-PHCs show similar electromagnetic fields in the interior cavities, due to the same size and interparticle spacing of Au NP cores. Au-PHCs show a much stronger electromagnetic field than Pt-PHCs surrounding the outer surface of the PHCs (Fig. 4a, b). It is expected that the plasmonic coupling between the spherical Au core and thin Au shell could be beneficial to the SERS analysis of absorbed reporters at the surface. We tested the SERS signal on Pt-PHCs, Pt-AHCs and Au-PHCs using 4-mercaptobenzoic acid (4-MBA) as a model reporter. 4-MBA shows two major bands at 1077 and $1587 \mathrm{~cm}^{-1}$, ascribed to the $v_{12}$ and $v_{8 \mathrm{a}}$ vibrations of the aromatic ring of MBA respectively [27]. The AHCs exhibit 3.2 times stronger enhancement than PHCs, because PHCs have a much weaker absorption than AHCs. While Au-PHCs show 6.6 and 2.1 times stronger enhancement than PHCs 


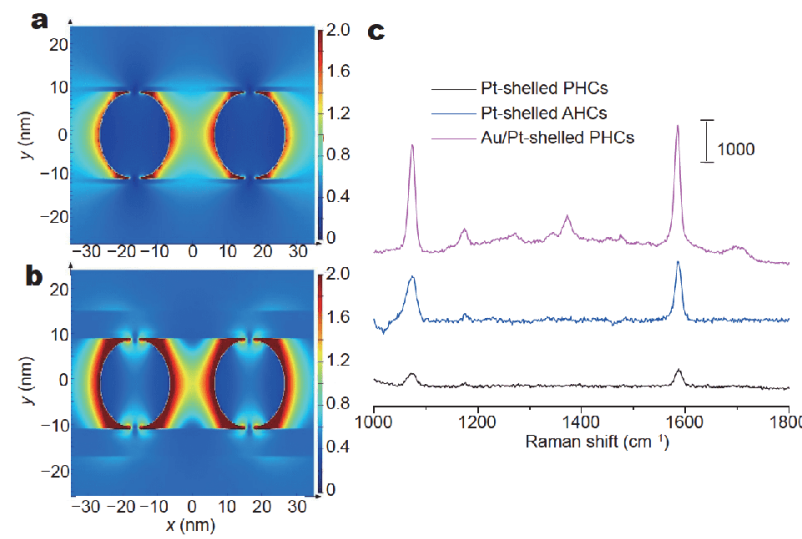

Figure 4 (a, b) FDTD simulation results showing the electromagnetic field distribution of (a) Pt-PHCs and (b) Au-PHCs respectively. (c) SERS spectra of 4-MBA with Pt-PHCs, Pt-AHCs and Au-PHCs.

and AHCs, respectively (Fig. 4c).

In conclusion, we have demonstrated controlled deposition of metals on polymer-NP HCs to synthesize AHCs or PHCs with metal shell periodically or thoroughly covering HCs. The selective metal deposition is strongly dependent on the selection of proper surfactants. These complex nanostructures have potential applications in plasmon-enhanced catalysis [28], sensing [29] and biomedicine [16].

\section{Received 23 February 2019; accepted 22 March 2019; published online 12 April 2019}

1 Liu Y, Goebl J, Yin Y. Templated synthesis of nanostructured materials. Chem Soc Rev, 2013, 42: 2610-2653

2 Sun Y, Xia Y. Shape-controlled synthesis of gold and silver nanoparticles. Science, 2002, 298: 2176-2179

3 Yu L, Yu XY, Lou XWD. The design and synthesis of hollow micro-/nanostructures: present and future trends. Adv Mater, 2018, 30: 1800939

4 Li A, Chang X, Huang Z, et al. Thin heterojunctions and spatially separated cocatalysts to simultaneously reduce bulk and surface recombination in photocatalysts. Angew Chem Int Ed, 2016, 55: 13734-13738

5 Huang Z, Raciti D, Yu S, et al. Synthesis of platinum nanotubes and nanorings via simultaneous metal alloying and etching. J Am Chem Soc, 2016, 138: 6332-6335

6 Nai J, Lou XWD. Hollow structures based on prussian blue and its analogs for electrochemical energy storage and conversion. Adv Mater, 2018, 37: 1706825

7 Yang Y, Liu J, Fu ZW, et al. Galvanic replacement-free deposition of $\mathrm{Au}$ on $\mathrm{Ag}$ for core-shell nanocubes with enhanced chemical stability and SERS activity. J Am Chem Soc, 2014, 136: 8153-8156

$8 \mathrm{Xu} \mathrm{H}$, Wang W. Template synthesis of multishelled $\mathrm{Cu}_{2} \mathrm{O}$ hollow spheres with a single-crystalline shell wall. Angew Chem Int Ed, 2007, 46: 1489-1492

9 Pang X, Zhao L, Han W, et al. A general and robust strategy for the synthesis of nearly monodisperse colloidal nanocrystals. Nat Na- notech, 2013, 8: 426-431

10 Sone ED, Zubarev ER, Stupp SI. Semiconductor nanohelices templated by supramolecular ribbons. Angew Chem Int Ed, 2002, 41: $1705-1709$

11 Zhang L, Roling LT, Wang X, et al. Platinum-based nanocages with subnanometer-thick walls and well-defined, controllable facets. Science, 2015, 349: 412-416

12 He J, Zhang P, Gong J, et al. Facile synthesis of functional Au nanopatches and nanocups. Chem Commun, 2012, 48: 7344

13 Gao C, Lu Z, Yin Y. Gram-scale synthesis of silica nanotubes with controlled aspect ratios by templating of nickel-hydrazine complex nanorods. Langmuir, 2011, 27: 12201-12208

14 Nie Z, Fava D, Kumacheva E, et al. Self-assembly of metal-polymer analogues of amphiphilic triblock copolymers. Nat Mater, 2007, 6: 609-614

15 Nie Z, Fava D, Rubinstein M, et al. "Supramolecular" assembly of gold nanorods end-terminated with polymer "pom-poms": effect of pom-pom structure on the association modes. J Am Chem Soc, 2008, 130: 3683-3689

16 Liu Y, He J, Yang K, et al. Folding up of gold nanoparticle strings into plasmonic vesicles for enhanced photoacoustic imaging. Angew Chem Int Ed, 2015, 54: 15916

17 Gao B, Arya G, Tao AR. Self-orienting nanocubes for the assembly of plasmonic nanojunctions. Nat Nanotech, 2012, 7: 433-437

18 Li W, Wang K, Zhang P, et al. Self-assembly of shaped nanoparticles into free-standing 2D and 3D superlattices. Small, 2016, 12: $499-505$

19 He J, Liu Y, Babu T, et al. Self-assembly of inorganic nanoparticle vesicles and tubules driven by tethered linear block copolymers. J Am Chem Soc, 2012, 134: 11342-11345

20 Stewart AF, Lee A, Ahmed A, et al. Rational design for the controlled aggregation of gold nanorods via phospholipid encapsulation for enhanced Raman scattering. ACS Nano, 2014, 8: 5462 5467

21 Huang Z, Liu Y, Zhang Q, et al. Collapsed polymer-directed synthesis of multicomponent coaxial-like nanostructures. Nat Commun, 2016, 7: 12147

22 Choueiri RM, Klinkova A, Thérien-Aubin $\mathrm{H}$, et al. Structural transitions in nanoparticle assemblies governed by competing nanoscale forces. J Am Chem Soc, 2013, 135: 10262-10265

23 Xiang Y, Wu X, Liu D, et al. Formation of rectangularly shaped Pd/ Au bimetallic nanorods: evidence for competing growth of the pd shell between the $\{110\}$ and $\{100\}$ side facets of Au nanorods. Nano Lett, 2006, 6: 2290-2294

24 Sun $\mathrm{H}, \mathrm{He} \mathrm{J}$, Wang $\mathrm{J}$, et al. Investigating the multiple roles of polyvinylpyrrolidone for a general methodology of oxide encapsulation. J Am Chem Soc, 2013, 135: 9099-9110

25 Graf C, Vossen DLJ, Imhof A, et al. A general method to coat colloidal particles with silica. Langmuir, 2003, 19: 6693-6700

26 Wang F, Cheng S, Bao Z, et al. Anisotropic overgrowth of metal heterostructures induced by a site-selective silica coating. Angew Chem Int Ed, 2013, 52: 10344-10348

27 Bishnoi SW, Rozell CJ, Levin CS, et al. All-optical nanoscale pH meter. Nano Lett, 2006, 6: 1687-1692

28 Aslam U, Rao VG, Chavez S, et al. Catalytic conversion of solar to chemical energy on plasmonic metal nanostructures. Nat Catal, 2018, 1: 656-665

29 Ding SY, Yi J, Li JF, et al. Nanostructure-based plasmon-enhanced Raman spectroscopy for surface analysis of materials. Nat Rev Mater, 2016, 1: 16021 
Acknowledgements This work was financially supported by the National Natural Science Foundation of China (21525626), the Program of Introducing Talents of Discipline to Universities (B06006), the Program for Professor of Special Appointment (Eastern Scholar) at Shanghai Institutions of Higher Learning, and the Startup Fund from Fudan University.

Author contributions Gong J and Nie $\mathrm{Z}$ conceived and supervised the research. Huang $\mathrm{Z}$ and Liu S performed the experiments; Zhang Y, Yang $\mathrm{J}$ and $\mathrm{Li} \mathrm{J}$ conducted SERS test and simulation. Huang $\mathrm{Z}$ wrote the paper with support from Nie $\mathrm{Z}$ and Gong J. All authors contributed to the general discussion.

Conflict of interest The authors declare no conflict of interest.

Supplementary information Experimental details and supporting figures are available in the online version of this paper.

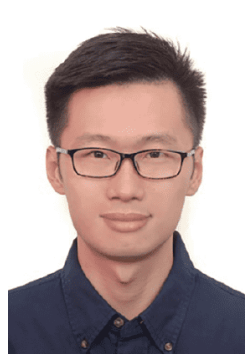

Zhiqi Huang obtained his $\mathrm{BSc}$ and $\mathrm{PhD}$ degree in chemical engineering from Tianjin University in 2011 and 2017, respectively, under the guidance of Prof. Jinlong Gong. From 2012 to 2015, he is a joint PhD student in Prof. Zhihong Nie's group at the University of Maryland, College Park (UMCP). He is currently a Postdoctoral Fellow at Nanyang Technological University with Prof. Hua Zhang. His research focuses on the synthesis and photo-/electro-catalytic applications of multicomponent nanomaterials.

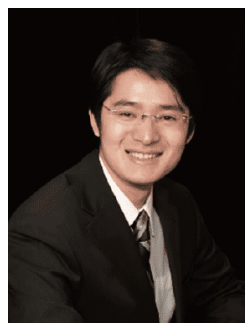

Zhihong Nie received his $\mathrm{PhD}$ degree in chemistry from the University of Toronto and worked as a NSERC Postdoctoral Fellow with Prof. George M. Whitesides at Harvard University. $\mathrm{He}$ is currently a Distinguished Professor in the Department of Macromolecular Science at Fudan University. Before that, he was an Associate Professor with tenure in the Department of Chemistry and Biochemistry at UMCP. He is the recipient of 3M Non-tenured Faculty Award, ACS PRF Doctoral New Investigator Award, NSF CAREER Award, etc. His research interests include molecular and nanoparticle self-assembly, biomedical imaging and delivery, programmable soft materials, and microfluidics.

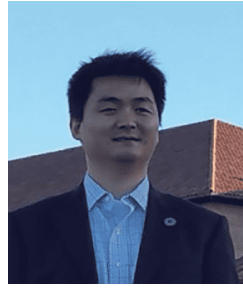

Jinlong Gong received his BSc and MSc degrees in chemical engineering from Tianjin University and his $\mathrm{PhD}$ degree from the University of Texas at Austin under the guidance of C. B. Mullins. After a stint with Prof. George M. Whitesides as a postdoctoral fellow at Harvard University, he joined the Faculty of Chemical Engineering at Tianjin University, where he currently holds a Cheung Kong Chair Professorship. He has served on the editorial boards for several journals including Chemical Society Reviews, Chemical Science, and AIChE Journal. He also serves as an Associate Editor for ACS Sustainable Chemistry \& Engineering. He is an elected Fellow of the Royal Society of Chemistry. He has published more than 200 peerrefereed papers and has been listed as a co-inventor on 73 patents and applications. His research interests include the catalytic conversions of green energy, utilization of carbon oxides, and synthesis and applications of nanostructured materials.

\section{金属在高分子-金纳米颗粒复合纳米链上的区域} 选择性生长

黄志琦 ${ }^{1}$, 刘思航 ${ }^{1}$ 张月皎 ${ }^{3}$, 杨晶亮 ${ }^{3}$, 李剑锋 ${ }^{3}$, 聂志鸿 $^{2^{*}}$, 巩金龙 ${ }^{1 *}$

摘要 模板法是可控合成复合纳米材料的一种重要手段. 然而, 纳 米材料在模板, 尤其是复合模板上的选择性生长仍面临巨大挑战. 本文以自组装聚乙烯-金纳米颗粒一维复合纳米链为软-硬复合模 板, 实现金属在该模板上可控地选择性沉积、生长. 通过选择恰当 的表面活性剂来调节金属生长的热力学, 金属可以选择性地生长 在金纳米颗粒的表面，得到金属间歇包覆的复合纳米链; 也可以同 时包覆在聚乙烯和金纳米颗粒的表面, 得到金属完全包覆的踠豆 荚状复合纳米链. 不同的包覆方式和包覆材料选择都会导致材料 光学性质的变化. 时间差分有限元模拟表明, 金包覆的豌豆荚状复 合纳米链的表面金壳层和内部金纳米颗粒之间存在极强的表面等 离子体耦合作用. 在表面增强拉曼实验中, 该复合纳米链表现出很 强的增强信号. 